\title{
Corrigendum to "Long Noncoding RNA TUG1/miR-29c Axis Affects Cell Proliferation, Invasion, and Migration in Human Pancreatic Cancer"
}

\author{
Yebin Lu, ${ }^{1}$ Ling Tang, ${ }^{2}$ Zhipeng Zhang, ${ }^{1}$ Shengyu Li, ${ }^{3}$ Shuai Liang, ${ }^{1}$ Liandong Ji, ${ }^{1}$ Bo Yang, \\ Yu Liu, ${ }^{4}$ and Wei Wei ${ }^{1}{ }^{1}$ \\ ${ }^{1}$ Department of General Surgery, Xiangya Hospital, Central South University, Changsha 410008, China \\ ${ }^{2}$ Department of Pharmacy, Xiangya Hospital, Central South University, Changsha 410008, China \\ ${ }^{3}$ Department of Vascular Surgery, Tianjin First Center Hospital, 300192, China \\ ${ }^{4}$ Department of Pathology, Hunan Provincial People's Hospital, Changsha 410005, China \\ Correspondence should be addressed to Wei Wei; csuweiwei2017@163.com
}

Received 26 February 2021; Accepted 26 February 2021; Published 5 July 2021

Copyright $\odot 2021$ Yebin Lu et al. This is an open access article distributed under the Creative Commons Attribution License, which permits unrestricted use, distribution, and reproduction in any medium, provided the original work is properly cited.

In the article titled "Long Noncoding RNA TUG1/miR-29c Axis Affects Cell Proliferation, Invasion, and Migration in Human Pancreatic Cancer" [1], BLASTn results [2] identified an error in the forward and reverse sequences of the TUG1 shRNA shown in Section 2.2 of the Materials and Methods Section. The reverse sequence of the TUG1 shRNA reported in the original article was identified as targeting the PMM2 gene sequence; however, the authors have confirmed that it was a mistake, and the correct sequences are as follows:

Correct forward sequence should read as follows:

$5^{\prime}$-gatccGCTTGGCTTCTATTCTGAATCCTTTCAAG AGAAGGATTCAGAATAGAAGCCAAGCTTTTTTG-3'.

Correct reverse sequence should read as follows:

5'-CAAAAAAGCTTGGCTTCTATTCTGAATCCTT

CTCTTGAAAGGATTCAGAATAGAAGCCAAGCG-3' .

\section{References}

[1] Y. Lu, L. Tang, Z. Zhang et al., "Long Noncoding RNA TUG1/miR-29c Axis Affects Cell Proliferation, Invasion, and Migration in Human Pancreatic Cancer," Disease Markers, vol. 2018, Article ID 6857042, 10 pages, 2018.

[2] C. Labbé, N. Grima, T. Gautier, B. Favier, and J. A. Byrne, "Semi-automated Fact-checking of Nucleotide Sequence Reagents in Biomedical Research Publications: The Seek \& Blastn tool," PLoS One, vol. 14, no. 3, Article ID e0213266, 2019. 